\title{
The increasing incidence of snowboard-related trauma
}

\author{
John R. Hayes and Jonathan I. Groner \\ The Trauma Program, Columbus Children's Hospital, and The Division of Pediatric Surgery, \\ Department of Surgery, The Ohio State University College of Medicine, Columbus, $\mathrm{OH} 43205$, \\ USA
}

\begin{abstract}
Purpose-To investigate injuries among children and adolescents who participate in downhill sports.

Methods-We collected trauma registry data (January 1999-May 2006) from a level 1 pediatric trauma center with an average snowfall of 28 in $(71 \mathrm{~cm}) / y$. Cases were analyzed for injury mechanism, injury type, organ injured, Injury Severity Score, age, sex, and whether or not an operation was required.
\end{abstract}

Results-There were 57 snowboarders and 22 skiers admitted during the study period. Forty-one (72\%) of snowboarders and 16 (73\%) of skiers required operations; $32(56 \%)$ of snowboarders and $9(41 \%)$ of skiers sustained fractures; and $14(25 \%)$ of snowboarders and $6(27 \%)$ of skiers sustained abdominal injuries. ( $P=\mathrm{NS}$ for all comparisons). Serious splenic injuries were more common in snowboarders (14\% vs 4\%), but the difference was not statistically significant. All skiing injuries occurred at recreational facilities (commercial skiing areas), whereas $12 \%$ of snowboard injuries occurred at home, other residence, or public parks $(P=.08)$. The most striking finding is the rising number of snowboarding injuries and the relatively stable rate of skiing injuries (see graph).

Conclusions-As the popularity of snowboarding rises, snowboarding injuries in children are increasing. Pediatric surgeons should be wary of the "snowboard spleen."

\section{Keywords}

Snowboard; Skiing; Recreation; Pediatric; Trauma

\begin{abstract}
Although downhill skiing is a well-established sport, snowboarding is relatively new: it was recognized by the International Olympic Committee in 1994 and premiered in the 1998 Winter Olympics in Nagano, Japan [1]. Snowboarding is remarkable for its dramatic rise in popularity [2] and its association with serious injuries [3]. The purpose of this study was to investigate skiing and snowboarding injuries at a level 1 pediatric trauma center that is located outside of the typical "snowbelt" in the United States.
\end{abstract}

\section{Methods}

This study was performed at a level 1 pediatric trauma center in Columbus, Ohio. The average snowfall in the region is 28 in $(71 \mathrm{~cm}) / \mathrm{y}$. We extracted data from a prospectively

(C) 2008 Elsevier Inc. All rights reserved

*Corresponding author. Tel.: +1 614722 3919; fax: +1 614722 3903. gronerj@ chi.osu.edu (J.I. Groner).

Presented at the 39th Annual Meeting of the Canadian Association of Pediatric Surgeons, August 23-26, 2007, St John's

Newfoundland, Canada. 
collected trauma registry data from January 1999 through May 2006 for all patients admitted for injuries that occurred while skiing or snowboarding. The trauma registry captures all patients admitted for the treatment of injury, including those who die in the emergency department after treatment. It does not capture patients discharged to home from the emergency department. By state policy, children less than 16 years old with major injuries are referred to pediatric trauma centers.

There were 79 cases that met the inclusion criteria. These cases were analyzed for injury mechanism, injury type, organ injured, Injury Severity Score, age, sex, and whether or not an operation was required. Statistical analysis was performed using $\chi^{2}$ (except where otherwise indicated), and a $P$ value of less than .05 was considered significant. This study was approved by the Institutional Review Board of Columbus Children's Hospital.

\section{Results}

There were 57 snowboarders and 22 skiers admitted during the study period. There were no deaths in either group. Only two snowboarders wore helmets and no skiers wore helmets. Snowboarders were more likely to be male than skiers ( $89 \%$ vs $68 \%, P<.05)$. Forty-one (72\%) snowboarders and 16 (73\%) skiers required operations; $32(56 \%)$ snowboarders and 9 (41\%) skiers sustained fractures; and $14(25 \%)$ snowboarders and $6(27 \%)$ skiers sustained abdominal injuries ( $P=\mathrm{NS}$ for all comparisons). Serious splenic injuries were more common in snowboarders than in skiers $(14 \%$ vs $4 \%)$, but the difference was not statistically significant. Skiers were hospitalized longer ( 4 vs 3 days, $P=.01$, Wilcoxon rank sum). All skiing injuries occurred at recreational facilities (commercial skiing areas), whereas $12 \%$ of snowboard injuries occurred in backyards or public parks (Table 1). The most striking finding is the rising number of snowboarding injuries and the relatively stable rate of skiing injuries (Fig. 1).

\section{Discussion}

Reports on downhill winter sports trauma often come from mountainous, high snowfall regions such as Norway [4,5] or Denver (average snowfall, 60 in [152 cm]/y) [6-8]. However, this study demonstrates that serious downhill sports injuries occur even in a region where the annual snowfall is low and ski resorts are few. The typical patient with downhill sports trauma in this study was white male snowboarder, but skiers had a longer length of stay. Other investigators have also found that male snowboarders are more likely to be injured (particularly splenic injuries), but have a shorter length of stay than skiers [3].

Downhill skiers, because of their cumbersome equipment, depend on chair lifts or similar devices at commercial ski resorts. Snowboarders, on the other hand, do not use poles, and their equipment is lightweight and easily carried. Thus snowboarders are able to practice their sport—and sustain injuries—outside of commercial skiing areas. This report appears to be the first to document serious injuries from "backyard" snowboarding.

More than one fourth of the children in this study sustained head trauma. Commercial ski resorts do not typically require helmet use, although head injuries are a common and potentially severe consequence of a skiing or snowboarding crash. In fact, head trauma is the leading cause of death among snowboarders and skiers [7]. Although other investigators found that snowboarders had 3-fold increase in head trauma compared to skiers [7], we found no difference in head injury rates in this study. However, because our study only captured injuries that required admission to a pediatric trauma center, patients with minor head injuries may have been omitted. 
Because the incidence of serious snowboard injuries is rising, there is a need for the development of injury prevention programs and awareness of downhill sports injury patterns. Mandating helmet use at resorts will not prevent all snowboard-related head injuries as some snowboarders are injured outside of commercial recreation areas. Fortunately, helmets are required for all international snowboarding competitions [1]. A broad public awareness campaign could promote the champion athletes who compete in these events as role models for younger downhill sports enthusiasts.

Finally, some investigators have named the snowboarding syndrome of male sex, aerial maneuvers (jumps), and splenic injury "boarder belly." [3] Our trauma center treated eight snowboarders with severe splenic fractures during the study period. Thus, our surgeons learned that snowboarders with symptoms of abdominal trauma—even if injured in backyards or parks-may have sustained "snowboard spleen."

\section{References}

1. Torjussen J, Bahr R. Injuries among elite snowboarders (FIS Snowboard World Cup). Br J Sports Med. 2006; 40:230-234. [PubMed: 16505079]

2. Sacco DE, Sartorelli DH, Vane DW. Evaluation of alpine skiing and snowboarding injury in a northeastern state. J Trauma. 1998; 44:654-659. [PubMed: 9555837]

3. Geddes R, Irish K. Boarder belly: splenic injuries resulting from ski and snowboarding accidents. Emerg Med Australas. 2005; 17:157-162. [PubMed: 15796731]

4. Brudvik C. Child injuries in Bergen, Norway. Injury. 2000; 31:761-767. [PubMed: 11154744]

5. Bergstrom KA, Askild O, Jorgensen NA, et al. Evaluation of skiing injuries by Injury Severity Score. Scand J Med Sci Sports. 1999; 9:110-113. [PubMed: 10220846]

6. Hagel BE, Pless IB, Hanley JA. Upper extremity snowboarding injuries: ten-year results from the Colorado Snowboard Injury Survey. Am J Sports Med. 2001; 29:676-678. [PubMed: 11573930]

7. Levy AS, Smith RH. Neurologic injuries in skiers and snowboarders. Semin Neurol. 2000; 20:233245. [PubMed: 10946744]

8. Prall JA, Winston KR, Brennan R. Severe snowboarding injuries. Injury. 1995; 26:539-542. [PubMed: 8550144] 


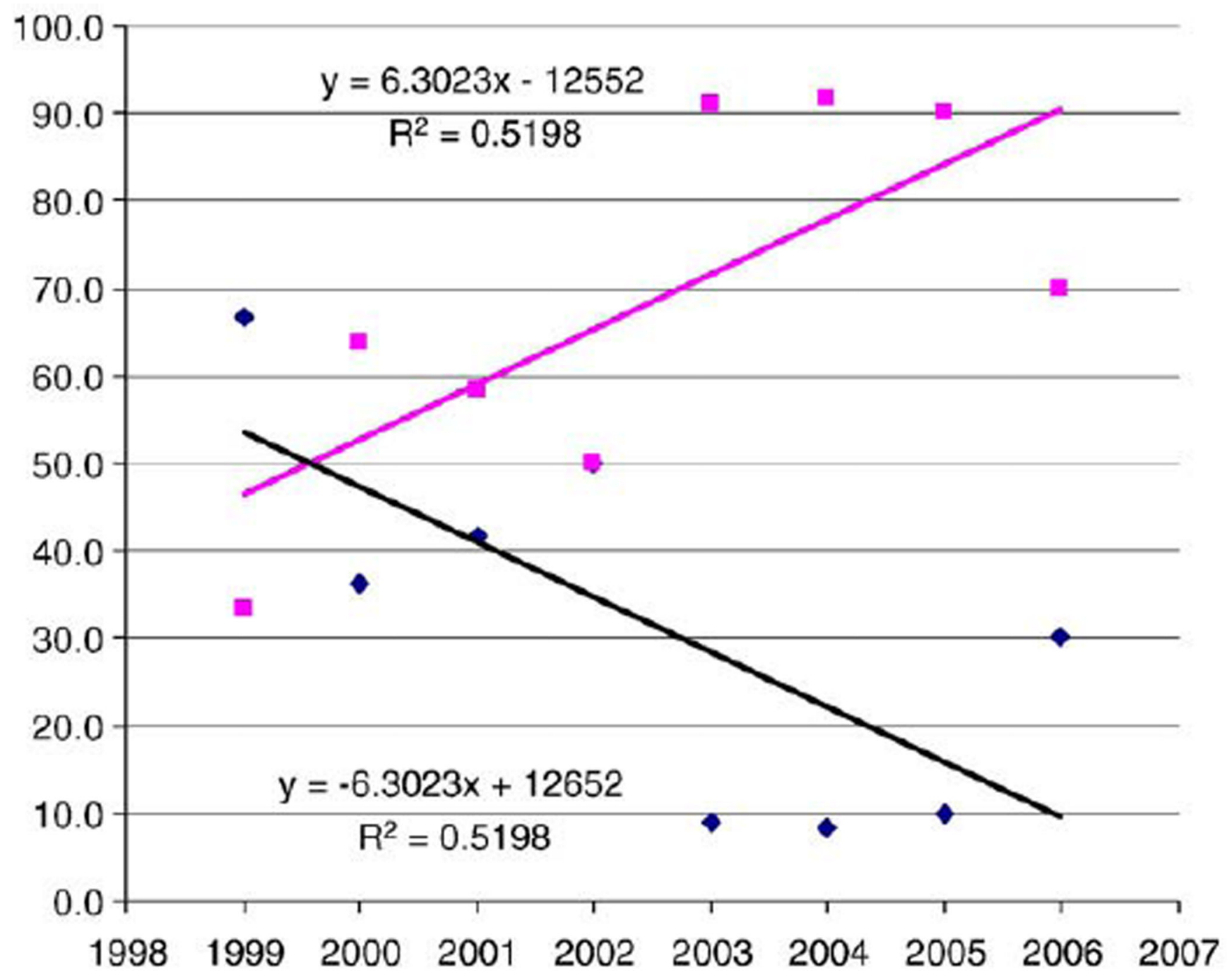

- Skiing - SnowBoard _L Linear (SnowBoard) — Linear (Skiing)

Fig. 1.

Ratio of skiers vs snowboarders (expressed as percent) admitted to the trauma service from 1999 to 2006. 
Table 1

Characteristics of injured skiers vs injured snowboarders

\begin{tabular}{|llll|}
\hline & Skiers & Snowboarders & $P$ value \\
\hline $\mathrm{n}$ & 22 & 57 & \\
Male sex & $15(68 \%)$ & $51(89 \%)$ & $.02^{*}$ \\
White & $18(82 \%)$ & $52(91 \%)$ & .24 \\
Median age & 13 & 14 & $.006^{* *}$ \\
Injured at commercial downhill sports facility & $22(100 \%)$ & $50(88 \%)$ & .08 \\
Head injury & $8(36 \%)$ & $14(25 \%)$ & .29 \\
Required operation & $16(73 \%)$ & $41(72 \%)$ & .94 \\
Fracture (any) & $9(41 \%)$ & $32(56 \%)$ & .22 \\
Abdominal injury & $6(27 \%)$ & $14(25 \%)$ & .80 \\
Splenic injury (severe) & $1(4 \%)$ & $8(14 \%)$ & .23 \\
Length of stay (d) & 4 & 3 & .01 \\
\hline
\end{tabular}

$P$ values calculated by $\chi^{2}$ except for median age and length of stay.

${ }^{*} P$ value statistically significant $\left(\chi^{2}\right)$.

${ }^{* *} P$ value statistically significant (Wilcoxon rank sum). 